Supporting Information for:

\title{
Path-DePendent Morphological Evolution of SE-TE Mesostructures PREPAREd By INORGANIC PHOTOTROPIC
}

\section{GROWTH}

\author{
KATHRYN R. HAMANN ${ }^{\dagger}$, AZHAR I. CARIM ${ }^{\dagger}$, MADELINE C. MEIER $^{\dagger}$, NATHAN S. LEWIS ${ }^{\dagger}, *$ \\ ${ }^{\dagger}$ Division of Chemistry and Chemical Engineering \\ California Institute of Technology \\ Pasadena, CA 91125
}




\section{S1. Contents}

This document contains a description of the experimental and modeling/simulation methods utilized in this work (Sections S2 and S3), additional scanning-electron micrographs (Section S4), Fourier transform data (Section S5), microstructural analysis data (Section S6), additional computer simulation data (Section S7), and a list of associated references (Section S8). 


\section{S2. Experimental Methods}

Materials and Chemicals $\mathrm{H}_{2} \mathrm{SO}_{4}$ (ACS Reagent, J. T. Baker), buffered HF improved etchant (Transene), $\mathrm{SeO}_{2}\left(99.4 \%\right.$, Alfa Aesar), and $\mathrm{TeO}_{2}(99+\%$, Sigma-Aldrich) were used as received. $\mathrm{H}_{2} \mathrm{O}$ with a resistivity $\geq 18.2 \mathrm{M} \Omega \mathrm{cm}$ (Barnstead Nanopure System) was used throughout. Au-coated $\mathrm{n}^{+}-\operatorname{Si}(100)(<0.005 \Omega \mathrm{cm}$, As-doped, $525 \pm 25 \mu \mathrm{m}$, single-side polished, Addison Engineering) was used as a substrate for deposition. Flash-Dry Ag Paint (SPI Supplies), EP21ARHTND Epoxy (MasterBond) and nitrocellulose-based nail polish were used to assemble the working electrodes.

Substrate Preparation $\mathrm{n}^{+}-\mathrm{Si}$ wafers were etched with buffered HF for $30 \mathrm{~s}$, rinsed with $\mathrm{H}_{2} \mathrm{O}$, dried under a stream of $\mathrm{N}_{2}(\mathrm{~g})$, and then immediately transferred to an electron-beam metal evaporator with a base pressure $<10^{-6}$ torr. Using an accelerating voltage of $10 \mathrm{kV}$, a $10 \mathrm{~nm} \mathrm{Ti}$ adhesion layer was deposited on the polished side of the wafer using a $40 \mathrm{~mA}$ deposition current and then $50 \mathrm{~nm}$ of Au was deposited on top of the Ti using a $150 \mathrm{~mA}$ deposition current. $20 \mathrm{~nm}$ of Ti was deposited on the unpolished side of the wafer to serve as a back-contact. The Au-topped Si sections were then cut into square $0.50 \mathrm{~cm}$ by $0.50 \mathrm{~cm}$ sections for use as deposition substrates.

Electrode Preparation Electrode assemblies were prepared by applying epoxy to the flat sides of each of two $\mathrm{Al}$ half-round bars ( 0.25 in diameter). The two bars were then joined together, with an $\sim 10 \mathrm{~mm}$ offset in the axial dimension to form a cylinder with two half-round ends. Polytetrafluoroethylene heat-shrink tubing was used to insulate the cylindrical section and epoxy was used to insulate the rounded side of one of the half-round ends. Ag paint was applied to the Ti-coated back surfaces of the Au-topped Si sections and the sections were affixed to the flat surface of the epoxied half-round end. Nail polish was used to insulate the remaining uncovered area on the flat surface that surrounded the Au-topped Si section. Figure S1 presents a schematic 
of an electrode assembly with an attached Au-topped Si section. Immediately before deposition, the surface of each electrode was briefly cleaned using a stream of $\mathrm{N}_{2}(\mathrm{~g})$.

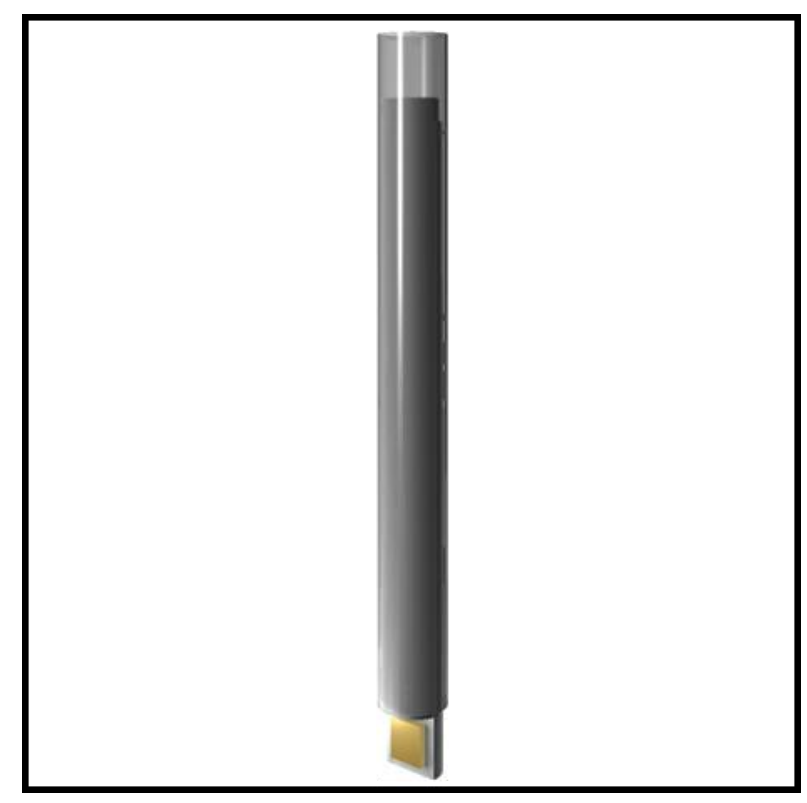

Figure S1. Schematic of an electrode assembly with an attached Au-topped Si section.

Electrode Illumination Illumination for photoelectrochemical growth was generated using two narrowband diode (LED) sources. The first (Thorlabs, M970L4) provided an intensityweighted average wavelength, $\lambda_{\text {avg }}$, value of $955 \mathrm{~nm}$ with a spectral bandwidth (FWHM) of $60 \mathrm{~nm}$. The second (Thorlabs, SOLIS-850C) was utilized in conjunction with a long pass filter with a 875

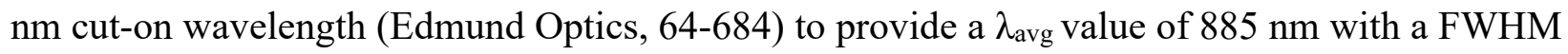
of $11 \mathrm{~nm}$. The $\lambda_{\text {avg }}=955 \mathrm{~nm}$ illumination was collected, condensed and collimated using a single aspheric lens $(\varnothing 50.8 \mathrm{~mm}, \mathrm{f}=32 \mathrm{~mm})$ whereas for $\lambda_{\text {avg }}=885 \mathrm{~nm}$ illumination an aspheric lens $(Ø 25.4 \mathrm{~mm}, \mathrm{f}=16 \mathrm{~mm})$ followed by two bi-convex lenses $(\varnothing 50.8 \mathrm{~mm}, \mathrm{f}=60 \mathrm{~mm}$ and $Ø 50.8 \mathrm{~mm}$, $\mathrm{f}=100 \mathrm{~mm}$ ) were utilized for illumination. A broadband film polarizer (LPNIRE200-B) was inserted after the lenses to effect vertical linear polarization. A 1500 grit ground-glass (UV Fused Silica) diffuser was placed immediately in front of the photoelectrochemical cell to ensure spatial homogeneity of the illumination. 
The light intensity incident on the electrode was measured by placing a calibrated $\mathrm{Si}$ photodiode (Thorlabs FDS100), instead of an electrode assembly, in the photoelectrochemical cell with electrolyte, and the steady-state current response of that Si photodiode was measured. Depositions with $\lambda_{\text {avg }}=885 \mathrm{~nm}$ and $955 \mathrm{~nm}$ were performed with light intensities of $I_{0}=110 \mathrm{~mW}$

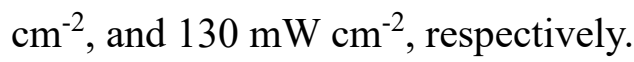

Photoelectrochemical Deposition Photoelectrochemical deposition was performed using a Bio-Logic SP-200 potentiostat. Deposition was performed in a two-compartment glass cell with a quartz window. A three-electrode configuration was utilized with an Ir wire counter electrode (99.999 \%, Sigma-Aldrich) isolated behind a porous glass frit and a $\mathrm{Ag} / \mathrm{AgCl}$ reference electrode (3.00 M KCl, Bioanalytical Systems). Films were deposited from an aqueous solution of 0.0200 $\mathrm{M} \mathrm{SeO}_{2}, 0.0100 \mathrm{M} \mathrm{TeO}_{2}$, and $2.00 \mathrm{M} \mathrm{H}_{2} \mathrm{SO}_{4}$. Deposition was effected by supplying a current density of $j=-8.00 \mathrm{~mA} \mathrm{~cm}^{-2}$ to the Au-coated electrode for $t_{0}=2.00 \mathrm{~min}$ (charge density of $Q_{0}=$ $0.96 \mathrm{mC} \mathrm{cm}^{-2}$ ), illuminated as detailed under the above subheading (Electrode Illumination). For depositions using two sequential, discrete illumination inputs, the electrode was transiently floated to open circuit following initial deposition as the illumination input was changed, and then deposition was continued with new illumination input by supplying $j=-8.00 \mathrm{~mA} \mathrm{~cm} \mathrm{~cm}^{-2}$ for an additional $t_{1}=t_{0}=2.00 \mathrm{~min}$ (additional charge density of $Q_{1}=Q_{0}=0.96 \mathrm{mC} \mathrm{cm}{ }^{-2}$ ) unless otherwise noted. After deposition, the electrode was immediately removed from the cell, rinsed with $\mathrm{H}_{2} \mathrm{O}$, and then dried under a stream of $\mathrm{N}_{2}(\mathrm{~g})$. The Au-coated substrate with top-facing Se-Te film was mechanically separated from the rest of the electrode assembly. The nitrocellulose-based insulation and the majority of the Ag paint were then removed mechanically.

Microscopy Scanning-electron micrographs (SEMs) were obtained with a FEI Nova NanoSEM 450 at an accelerating voltage of $5.00 \mathrm{kV}$ with a working distance of $5 \mathrm{~mm}$ and an in- 
lens secondary electron detector. Micrographs obtained for quantitative analysis were acquired with a resolution of 172 pixels $\mu \mathrm{m}^{-1}$ over $\sim 120 \mu \mathrm{m}^{2}$ areas. Micrographs that were used to produce display figures were acquired with a resolution of 344 pixels $\mu \mathrm{m}^{-1}$ over $\sim 2 \mu \mathrm{m}^{2}$ areas.

Raman Spectroscopy Raman spectra were collected with a Renishaw inVia Raman microprobe equipped with a Leica DM 2500 M microscope, a Leica N Plan 50x objective (numerical aperture $=0.75$ ), an 1800 lines $\mathrm{mm}^{-1}$ grating, and a CCD detector configured in a $180^{\circ}$ backscatter geometry. A $532 \mathrm{~nm}$ diode-pumped solid-state (DPSS) laser (Renishaw RL532C50) was used as the excitation source and a $10 \mu \mathrm{W}$ radiant flux was incident on the surface of the sample. A $\lambda / 4$ plate was used to circularly polarize the incident excitation. No polarizing collection optic was used.

X-Ray Diffraction X-ray diffraction (XRD) data was collected using a Bruker D8 Discover diffractometer with a $\mathrm{Cu} \mathrm{K} \alpha$ source and a two-dimensional detector in a Bragg-Brentano geometry. 


\section{S3. Modeling and Simulation Methods}

Simulation of Film Morphology The growths of the photoelectrochemically deposited films were simulated with an iterative growth model in which electromagnetic simulations were first used to calculate the local photocarrier-generation rates at the film surface. Then, mass addition was simulated via a Monte Carlo method wherein the local photocarrier-generation rate weighted the local rate of mass addition along the film surface.

Growth simulations began with a bare, semi-infinite planar substrate. In the first step, the light-absorption profile under a linearly polarized, plane-wave illumination source was calculated using full-wave finite-difference time-domain (FDTD) simulations ("FDTD Solutions" software package, Lumerical) with periodic boundary conditions along the substrate interface. In the second step, a Monte Carlo simulation was performed in which an amount of mass, equaling that of a 5 $\mathrm{nm}$ planar layer covering the simulation area, was added to the upper surface of the structure with a probability $F$ :

$$
F(G)=G \prod_{i=1}^{3} \frac{x_{i}}{r_{i}} \quad \text { (Equation 1) }
$$

where $G$ is the spatially dependent photocarrier-generation rate at the deposit/solution interface, $x_{i}$ is the fraction of $\mathrm{i}^{\text {th }}$ nearest neighbors occupied in the cubic lattice, and $r_{i}$ is the distance to the $\mathrm{i}^{\text {th }}$ nearest neighbor. The multiplicative sum in the definition of this probability (Equation 1) serves to reduce the surface roughness of the film so as to mimic the experimentally observed surface roughness. After the initial Monte Carlo simulation, the absorbance of the new, structured film was then calculated in the same manner as for the initial planar film, and an additional Monte Carlo simulation of mass addition was performed. This process of absorbance calculation and mass addition was repeated until the simulated morphologies achieved heights equivalent to those exhibited by the experimentally generated deposits. To model growth using two sequential, 
discrete illumination inputs, the computational process was also first iterated using the initial illumination input until the simulated morphology height achieved heights equivalent to those exhibited by the experimentally generated deposits using that initial input. Then, the simulated illumination was updated to represent the new optical input and the computational process was further iterated until the simulated morphologies achieved heights equivalent to those exhibited by the experimentally generated deposits for growth with sequential, discrete illumination inputs.

Previously measured values of the wavelength-dependent complex refractive index of SeTe were utilized. A value of $n=1.33$ was used as the refractive index of the electrolyte, regardless of wavelength. ${ }^{1}$ Simulations of the film morphology utilized the intensity-weighted average wavelengths, $\lambda_{\text {avg }}$, of the experimental sources described in Section S2. A two-dimensional square mesh with a lattice constant of $5 \mathrm{~nm}$ was used for the simulations.

Dipole Simulations Dipole simulations were performed using a FDTD method. A twodimensional square simulation plane was utilized. Dipoles were arranged with a separation of twice the emission wavelength and the oscillation axis was set perpendicular to the separation axis. 


\section{S4. Additional Scanning-Electron Micrographs}

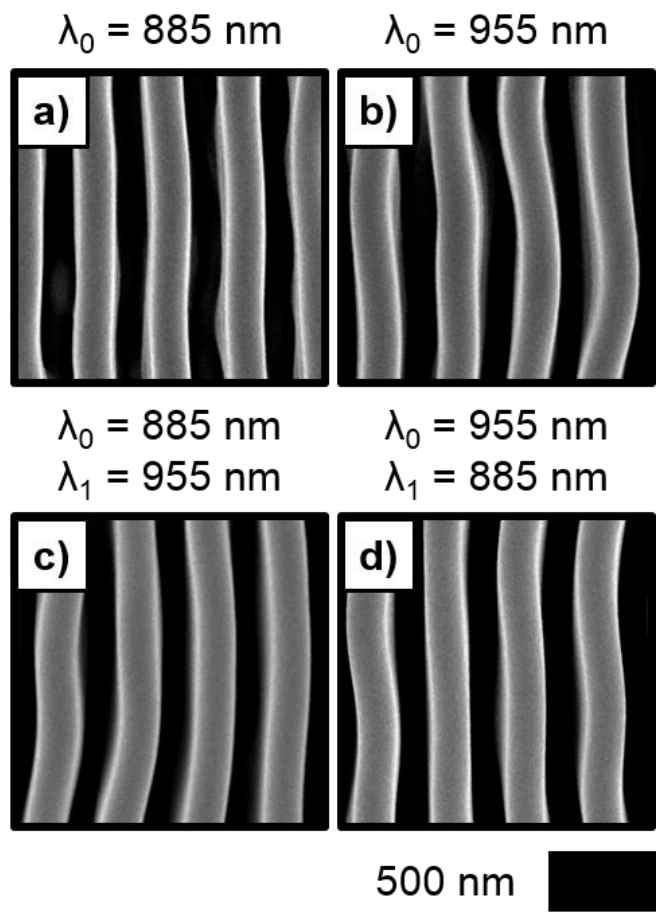

Figure S2. Top-down SEMs representative of films generated with the indicated $\lambda_{0}$ illumination (a) and (b) and then extended with the indicated $\lambda_{1}$ illumination (c) and (d).

$$
\begin{array}{ll}
\lambda_{0}=885 \mathrm{~nm} & \lambda_{0}=955 \mathrm{~nm} \\
\lambda_{1}=955 \mathrm{~nm} & \lambda_{1}=885 \mathrm{~nm}
\end{array}
$$

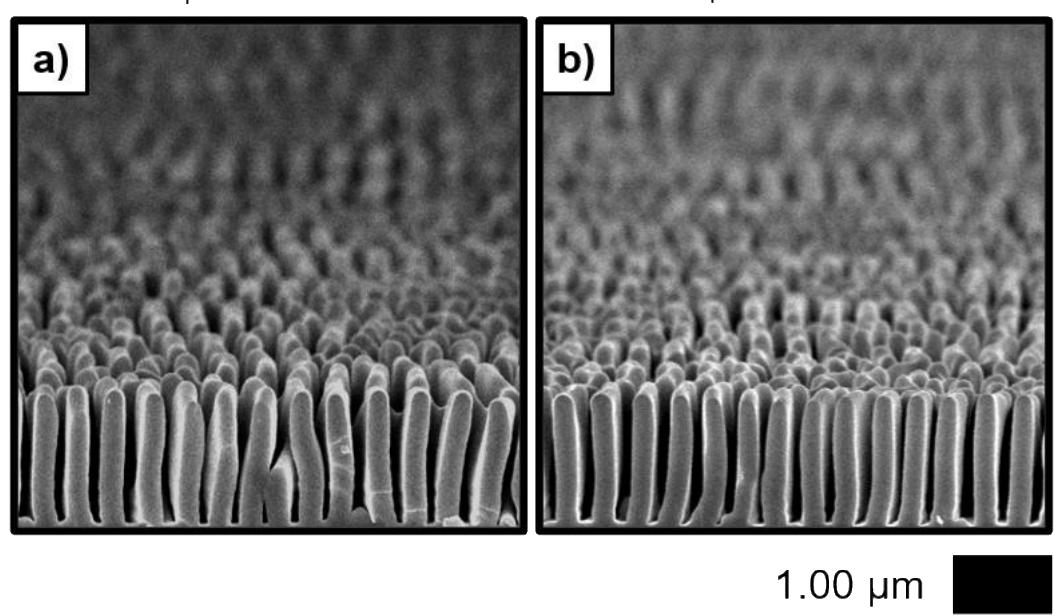

Figure S3. Representative cross-sectional, intermediate magnification SEMs of deposits generated first with the indicated $\lambda_{0}$ illumination and then extended with the indicated $\lambda_{1}$ illumination. 


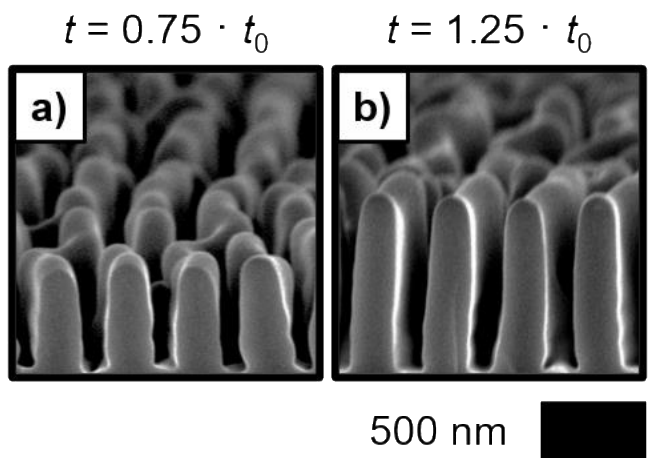

Figure S4. Representative cross-sectional SEMs of deposits generated using $\lambda=955 \mathrm{~nm}$ illumination with an intensity of $I=I_{0}$ for the indicated time $\left(j=j_{0}\right) . Q=0.75 \cdot Q_{0}$ for (a) and $1.25 \cdot Q_{0}$ for (b).

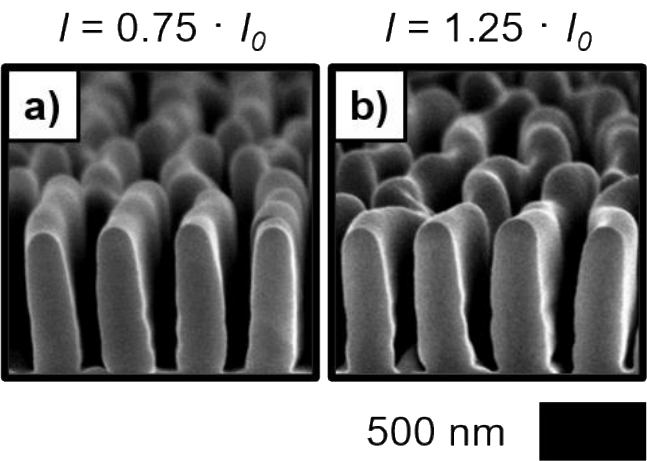

Figure S5. Representative cross-sectional SEMs of deposits generated using $\lambda=955 \mathrm{~nm}$ illumination with the indicated intensity for $t=t_{0}\left(j=j_{0} ; Q=Q_{0}\right)$. 
S5. Fourier Transform Data

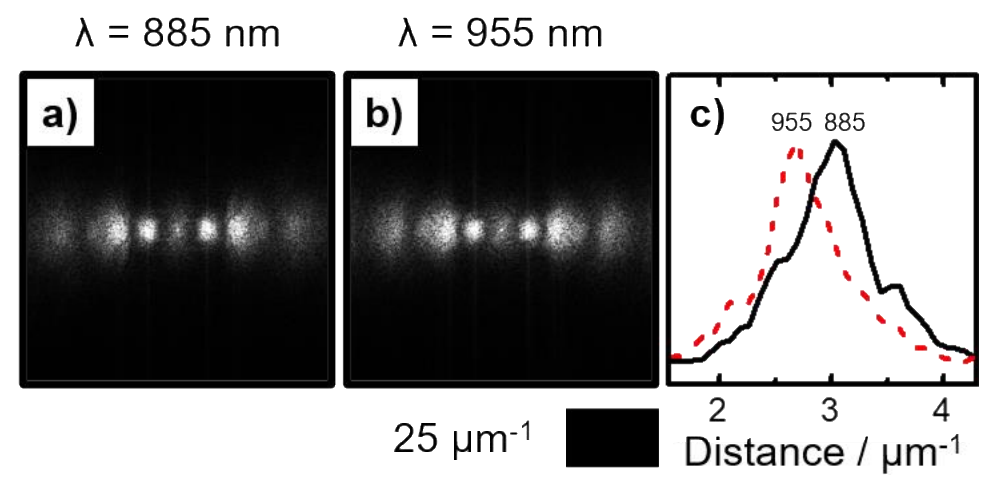

Figure S6. (a) and (b) 2D FTs derived from top-down SEM data of deposits generated with the indicated $\lambda$ illumination. (c) Fourier spectra derived from the 2D FT data in (a) and (b). 


\section{S6. Deposit Microstructural Analysis}

Figure S7 presents a representative Raman spectrum of a deposit generated via inorganic phototropic growth. The spectrum exhibits two principal modes centered at $144 \mathrm{~cm}^{-1}$ and $175 \mathrm{~cm}^{-}$

1. These modes are consistent with a substitutional alloy of Se and Te in a trigonal crystal structure common to each element in the pure phase. ${ }^{2}$ Figure S8 presents a representative X-ray diffraction (XRD) data of a deposit generated via inorganic phototropic growth. The observed reflections are consistent with the crystalline phase suggested by the Raman analysis and indicate a polycrystalline character and lack of preferred orientation. ${ }^{3,4}$

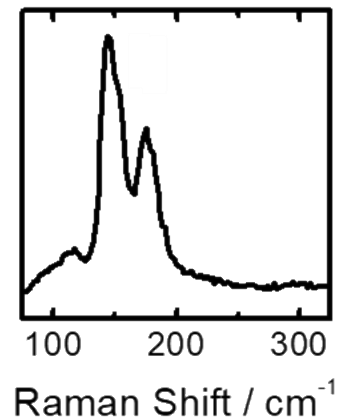

Figure S7. Representative Raman spectrum of a deposit generated via inorganic phototropic growth.

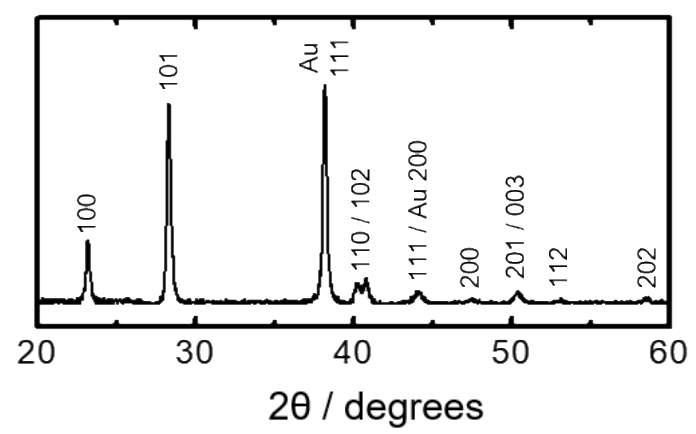

Figure S8. Representative XRD data of a deposit generated via inorganic phototropic growth with reflections characteristic of Se-Te and the Au substrate indicated. 


\section{S7. Additional Computer Simulation Data}

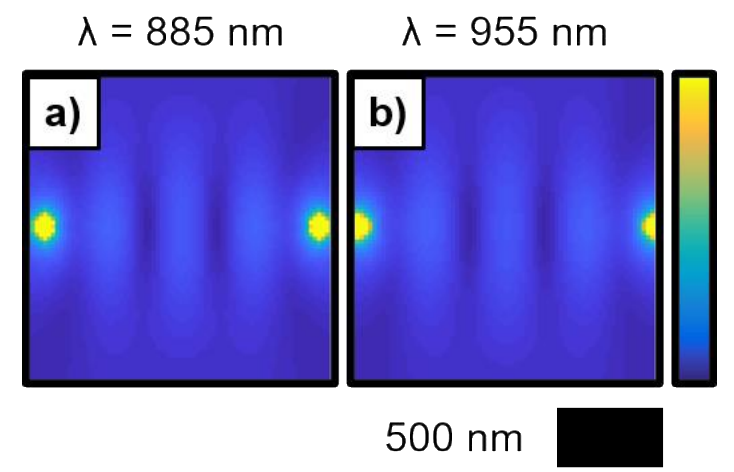

Figure S9. Normalized time-averages of the E-field magnitude from two dipoles emitting radiation with the indicated free space wavelength in a medium of refractive index $n=1.33$. Dipoles separated by a distance of twice the wavelength along the horizontal with the oscillation axis perpendicular to the axis of separation.

Point dipole sources were used to model the amplitude of the electric field modulation at the growth interface caused by scattering of the incident illumination by the by the topology of the nascent electrochemically generated deposit. Figure S3a and b present simulations of normalized time-averaged electric field magnitudes from two dipoles emitting radiation with free space wavelengths of $\lambda=885 \mathrm{~nm}$ and $955 \mathrm{~nm}$, respectively, in a medium of index $n=1.33$ (reflective of the growth electrolyte) and separated by a distance of two wavelengths perpendicular to the oscillation axis. In each case, periodic bands of elevated field intensity were observed. The spacing and width of these regions of elevated field intensity were proportional to the wavelength; periods of $333 \mathrm{~nm}$ and $359 \mathrm{~nm}$ were observed for $\lambda=885 \mathrm{~nm}$ and $955 \mathrm{~nm}$, respectively. These data agree with the experimental observation of scaling of lamellar pitch with the input $\lambda_{\text {avg. }}$ This behavior is consistent with the notion that photoelectrochemical growth occurs preferentially in areas of localized field elevation and thereby physically records the input illumination. 


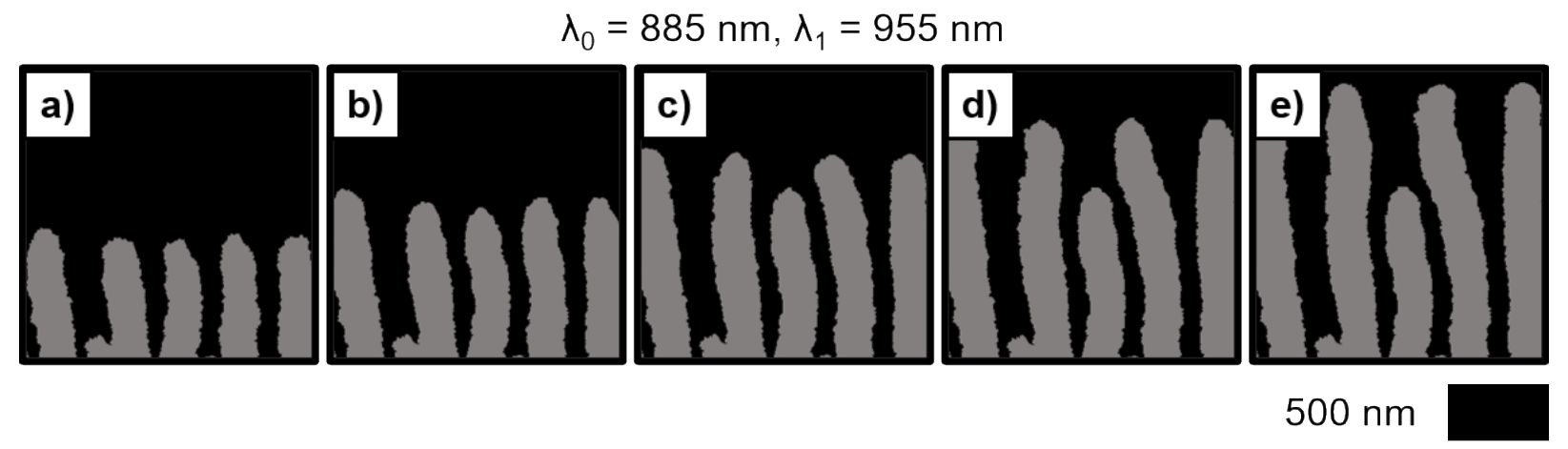

Figure S10. From left to right: successive iterations of simulated morphologies generated initially generated using $\lambda_{0}$ $=885 \mathrm{~nm}$ illumination and progressively extended the indicated $\lambda_{0}$ illumination and progressively extended with $\lambda_{1}=$ $955 \mathrm{~nm}$ illumination.

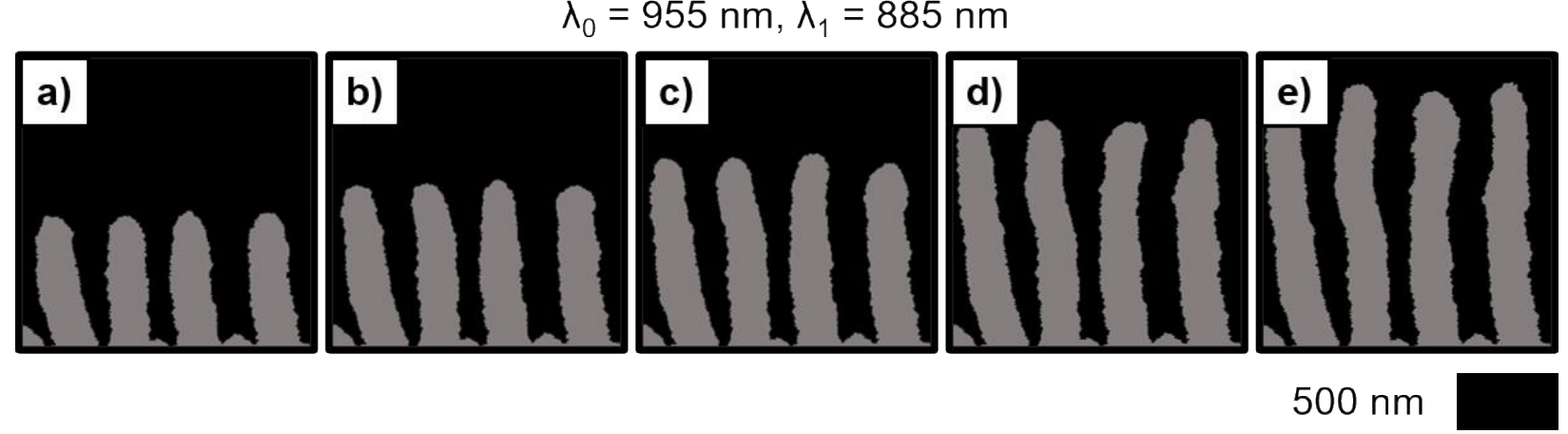

Figure S11. From left to right: successive iterations of simulated morphologies generated initially generated using $\lambda_{0}$ $=955 \mathrm{~nm}$ illumination and progressively extended the indicated $\lambda_{0}$ illumination and progressively extended with $\lambda_{1}=$ $885 \mathrm{~nm}$ illumination. 


\section{S8. References}

(1) Hale, G. M.; Querry, M. R. Optical Constants of Water in the 200-nm to 200- $\mu \mathrm{m}$ Wavelength Region. Appl. Opt. 1973, 12, 555-563.

(2) Geick, R.; Steigmeier, E. F.; Auderset, H. Raman Effect in Selenium-Tellurium Mixed Crystals. Phys. Status Solidi B 1972, 54, 623-630.

(3) Smith, T. W.; Smith, S. D.; Badesha, S. S. Chemical Alloying, a Novel Method for the Preparation of Homogenous $\mathrm{Se}_{\mathrm{x}} \mathrm{Te}_{1-\mathrm{x}}$ Alloys. J. Am. Chem. Soc. 1984, 106, 7247-7248.

(4) Mayers, B.; Gates, B.; Yin, Y.; Xia, Y. Large-Scale Synthesis of Monodisperse Nanorods of Se/Te Alloys Through a Homogenous Nucleation and Solution Growth Process. Adv. Mater. 2001, 13, 1380-1384. 